\title{
Isolated Tuberculosis liver abscess in an immunocompetent patient.
}

\author{
yazan sallam ${ }^{1}$, Dr. Ramsey Jasim ${ }^{1}$, Nabeel Qasem ${ }^{1}$, and Mohammad Kloub ${ }^{1}$ \\ ${ }^{1}$ Hamad Medical Corporation
}

September 25, 2021

\begin{abstract}
Tuberculous (TB) infection remains one of the leading causes of death, especially in developing countries. TB liver involvement is a rare extrapulmonary manifestation of TB and is usually found as a secondary involvement to TB of the lung. Here we are presenting a case of Isolated TB liver abscess.
\end{abstract}

\section{Introduction:}

Globally, an estimated 10.0 million people fell ill with (TB) in 2019. TB is a communicable disease that is a major health concern around the world, one of the top 10 causes of death globally and the leading cause of death from an infectious agent. ${ }^{1}$ (TLA) without active pulmonary or military TB is a rare entity. ${ }^{2}$ Patients usually present with non-specific symptoms, which makes it difficult to diagnose. Here we present a case of an isolated TLA in an immunocompetent patient, in which the patient had symptoms of abdominal pain, vomiting, and fever, with no specific findings to suggest TLA on radiological imaging.

\section{Case presentation :}

A 62-year-old male patient presented to our hospital, complaining of a fever of 10 days duration.

This patient is a known case of CKD stage 5, on regular follow-ups, was doing relatively fine when he started having fever for 10 days before presenting to our hospital, the fever was mainly at night, documented at about 38 degrees, associated with chills and rigors, and occasional vomiting.

On physical examination, except for right upper quadrant tenderness, he had no other physical exam findings.

Labs showed: hemoglobin $9.0 \mathrm{gm} / \mathrm{dl}$, White blood cells 24000/ $\mathrm{mm}^{3}$ (neutrophils $88 \%$ ), platelets count 322,000 , Blood urea nitrogen $39 \mathrm{mmol} / \mathrm{L}$ (high) , Creatinine $567 \mathrm{umol} / \mathrm{L}$ (this is his baseline kidney function), C- Reactive protein $136 \mathrm{mg} / \mathrm{L}$ (high), Total Bilirubin $21 \mathrm{umol} / \mathrm{L}$ (normal range up to 22), elevated liver enzymes, Alkaline phosphatase $514 \mathrm{U} / \mathrm{L}$ (normal range up to 129).

The patient was investigated for Hepatitis B, Hepatitis C, and HIV and all were negative, Chest X-ray was normal, routine and microscopic examination of stool showed no cyst or ova; amoebic serology was negative.

US Abdomen was done which showed Ill-defined heterogenous structure noted at the right lobe liver, so MRI abdomen was done for the patient, without contrast, which showed Focal hepatic lesion in segment VIII/IVb (4.8 cm in maximal dimension), Enlarged abdominal lymph nodes, including portocaval lymph nodes which are compressing the mid-CBD and causing upstream biliary dilatation (Figure 1).

A

Figure 1 (A): Axial MRI section showing liver abscess. (B): MRI Sagittal view showing liver abscess Figure 1 (A): Axial $]$ 
US-guided aspiration was attempted, but the content was very thick that nothing was aspirated, so eight liver biopsies were taken from the right liver lobe lesion using a coaxial 18/16 G biopsy system.

The biopsies were sent for Ziehl-Neelsen (ZN) stain, acid-fast bacilli culture, polymerase chain reaction (PCR) for TB, and other routine microbiological investigations alongside histopathology.

TB PCR came positive the next day, histopathology showed necrotizing granulomatous inflammation. Diagnosis of TLA was made, and the patient was started on Anti-TB medications (isoniazid 300mg daily, rifampin 600mg daily, pyrazinamide 1750mg Q48 hours, ethambutol 800mg Q48 hours. After 3 days of starting the treatment, the patient started to improve clinically, evidenced by the absence of fever, gradual improvement of the abdominal pain and vomiting, and decrease in inflammatory markers.

The patient was discharged after 1 week from starting treatment, with regular follow-ups scheduled, he came back to the Infectious disease clinic after 1 month, with complete resolution of GI symptoms, and no recurrent fever, repeat US was not done as the patient was clinically improving.

\section{Discussion:}

TB infection remains one of the leading causes of death, especially in developing countries. ${ }^{3}$ It is extremely important for clinicians to understand the nature of the disease and its wide variety of presentations, in addition to having a high index of suspension when dealing with patients in areas where TB remains an endemic infection.

In extra-pulmonary TB, liver tuberculosis has been described as a rare entity, but not an exceptional one. Isolated TLA is an extremely rare condition, The incidence of which was found to be $0.34 \% .{ }^{6}$ It is often misdiagnosed as a pyogenic or amoebic abscess. ${ }^{4}$ Most of the cases in the literature occur alongside miliary TB of the lungs, which spread to the liver by hematogenous spread. ${ }^{5}$

The diagnosis of TLA has always been challenging as the symptoms of this condition are not specific. ${ }^{7}$ Patients usually present with constitutional symptoms like fever, anorexia, and weight loss. As there are no specific symptoms, signs, or lab investigations for TLA, diagnosis depends on a high index of suspicion, especially in patients coming from endemic areas for TB. Radiological imaging modalities are usually not helpful in differentiating between pyogenic, amoebic, or TB liver abscess. ${ }^{8}$ In our case, our patient came with vague symptoms, which manifested mainly as fever and abdominal pain.

The diagnosis of TLA requires the use of ZN stains, acid-fast bacilli culture and PCR on the specimen collected. ${ }^{9}$ In liver biopsies, granuloma formation can be seen in around $80 \%$ to $100 \%$ of cases; and caseation in up to $83 \% .{ }^{10} \mathrm{PCR}$ assays are positive in up to $88 \%$ of TLA cases. ${ }^{11}$ No specific lab investigations can help diagnose TLA, with previous studies showing elevated Alkaline phosphatase as the most frequent finding. ${ }^{12}$

Medical treatment for TB is a debatable subject, with most centers recommending treatment with Quadruple therapy for 1 year. ${ }^{13}$ In our case, treatment with Anti- TB medication was started for the patient and he started showing improvement within 1 week of starting the medications, and he was seen 1 month after discharge with complete resolution of the fever and his previous symptoms.

\section{Conclusion:}

Isolated TLA is an extremely rare condition, but should always be considered in a patient presented with liver abscess, especially from an endemic area. There are no specific symptoms or signs for TLA, and radiological investigations usually do not help differentiate between TLA and other causes of liver abscess. Treatment with quadruple therapy is the mainstay for treatment, and the prognosis is usually good.

\section{Statements:}

Acknowledgment:

Authors would like to acknowledge the internal medicine department at Hamad Medical Corporation for supporting this publication. 
Statement of Ethics:

Written informed consent was obtained from the patient to allow the publication of information including images.

Case approved by HMC Medical Research Center.

Disclosure Statement:

The authors report no conflicts of interest in this work.

Funding sources

Qatar National Library.

Authors contribution:

All authors contributed equally in writing and editing.

References:

1. GLOBAL TUBERCULOSIS REPORT 2020. Published online 2020. Accessed August 23, 2021. http://apps.who.int/bookorders.

2. Dey J, Gautam H, Venugopal S, et al. Tuberculosis as an Etiological Factor in Liver Abscess in Adults. Tuberc Res Treat . 2016;2016:1-4. doi:10.1155/2016/8479456

3. Bhargava A, Bhargava M. Tuberculosis deaths are predictable and preventable: Comprehensive assessment and clinical care is the key.J Clin Tuberc Other Mycobact Dis . Published online 2020. doi:10.1016/j.jctube.2020.100155

4. Rizwan ASM, Islam MR, Yusuf MG. Isolated tuberculous liver abscess in an immunocompromised adult: A case report. Bangladesh J Med Sci . Published online 2018. doi:10.3329/bjms.v17i1.35298

5. Saturinas LESR, Hernandez EA. A case report of an uncommon extra-pulmonary tuberculosis presenting as an isolated tuberculous liver abscess in a 63-year-old immunocompetent male. Phillippine $J$ Intern Med . Published online 2020.

6. Essop AR, Segal I, Posen J, Noormohamed N, Palhologisl NOORMOHAMED SN, Medical Officer S. Tuberculous abscess of the liver A case report.SA Med J . 1983;63.

7. Krishna Nalabothu S, Menon SK. Scholars Journal of Medical Case Reports ISSN 2347-9507 (Print) Tuberculous liver abscess-An unusual presentation. Sch J Med Case Rep . 2014;2(10):687-689. Accessed August 23, 2021. http://saspjournals.com/sjmcr

8. Schininà V, Albarello F, Cristofaro M, et al. Diagnostic imaging of hepatic tuberculosis: Case series. Int J Tuberc Lung Dis . Published online 2018. doi:10.5588/ijtld.17.0710

9. Ruiz L, Maya MA, Rueda ZV, López L, Vélez LA. Current characteristics of tuberculosis and human immunodeficiency virus co-infection in a cohort of hospitalized patients in Medellín, Colombia. Biomedica . Published online 2018. doi:10.7705/BIOMEDICA.V38I3.3862

10. de Mello ES, Ferreira Alves VA. Hepatic Granulomas: Differential Diagnosis. Pract Hepatic Pathol A Diagnostic Approach Second Ed . Published online January 1, 2018:289-300. doi:10.1016/B978-0-323-428736.00019-6

11. Chaudhary P. Hepatobiliary tuberculosis. Ann Gastroenterol Q Publ Hell Soc Gastroenterol . 2014;27(3):207. Accessed August 23, 2021. /pmc/articles/PMC4073015/

12. SW H, YJ K, EJ C, et al. [Clinical features of hepatic tuberculosis in biopsy-proven cases]. Korean $J$ Hepatol . 2009;15(2):159-167. doi:10.3350/KJHEP.2009.15.2.159 
13. Baveja C, Gumma V, Chaudhary M, Jha H. Primary tubercular liver abscess in an immunocompetent adult: a case report. J Med Case Reports 200931 . 2009;3(1):1-4. doi:10.1186/1752-1947-3-78

\section{Hosted file}

Figures.docx available at https://authorea.com/users/437651/articles/539138-isolatedtuberculosis-liver-abscess-in-an-immunocompetent-patient 